\title{
A new application of quasi power increasing sequences. I
}

Hüseyin Bor ${ }^{*}$

\section{"Correspondence:}

hbor33@gmail.com

P.O. Box 121, Bahçelievler, Ankara,

TR-06502, Turkey

\begin{abstract}
In (Rocky Mt. J. Math. 38:801-807, 2008), we proved a theorem dealing with an application of quasi- $\sigma$-power increasing sequences. In the present paper, we prove that theorem under less and more weaker conditions. This theorem also includes some new and known results.
\end{abstract}

MSC: 40D15; 40F05; 40G99; 46A45

Keywords: Hölder's inequality; sequence spaces; absolute summability; increasing sequences

\section{Introduction}

A positive sequence $\left(b_{n}\right)$ is said to be almost increasing if there exists a positive increasing sequence $c_{n}$ and two positive constants $A$ and $B$ such that $A c_{n} \leq b_{n} \leq B c_{n}$ (see [1]). A sequence $\left(\lambda_{n}\right)$ is said to be of bounded variation, denoted by $\left(\lambda_{n}\right) \in \mathcal{B V}$, if $\sum_{n=1}^{\infty}\left|\Delta \lambda_{n}\right|=$ $\sum_{n=1}^{\infty}\left|\lambda_{n}-\lambda_{n+1}\right|<\infty$. A positive sequence $X=\left(X_{n}\right)$ is said to be a quasi- $\sigma$-power increasing sequence if there exists a constant $K=K(\sigma, X) \geq 1$ such that $K n^{\sigma} X_{n} \geq m^{\sigma} X_{m}$ holds for all $n \geq m \geq 1$. It should be noted that every almost increasing sequence is a quasi- $\sigma$ power increasing sequence for any nonnegative $\sigma$, but the converse may not be true as can be seen by taking an example, say $X_{n}=n^{-\sigma}$ for $\sigma>0$ (see [2]). Let $\left(\varphi_{n}\right)$ be a sequence of complex numbers and let $\sum a_{n}$ be a given infinite series with partial sums $\left(s_{n}\right)$. We denote by $z_{n}^{\alpha}$ and $t_{n}^{\alpha}$ the $n$th Cesàro means of order $\alpha$, with $\alpha>-1$, of the sequences $\left(s_{n}\right)$ and $\left(n a_{n}\right)$, respectively, that is,

$$
\begin{aligned}
& z_{n}^{\alpha}=\frac{1}{A_{n}^{\alpha}} \sum_{v=0}^{n} A_{n-v}^{\alpha-1} s_{v}, \\
& t_{n}^{\alpha}=\frac{1}{A_{n}^{\alpha}} \sum_{v=1}^{n} A_{n-v}^{\alpha-1} v a_{v},
\end{aligned}
$$

where

$$
A_{n}^{\alpha}=\left(\begin{array}{c}
n+\alpha \\
n
\end{array}\right)=\frac{(\alpha+1)(\alpha+2) \cdots(\alpha+n)}{n !}=O\left(n^{\alpha}\right), \quad A_{-n}^{\alpha}=0 \quad \text { for } n>0 .
$$

The series $\sum a_{n}$ is said to be summable $\varphi-|C, \alpha|_{k}, k \geq 1$ and $\alpha>-1$, if (see [3])

$$
\sum_{n=1}^{\infty}\left|\varphi_{n}\left(z_{n}^{\alpha}-z_{n-1}^{\alpha}\right)\right|^{k}=\sum_{n=1}^{\infty} n^{-k}\left|\varphi_{n} t_{n}^{\alpha}\right|^{k}<\infty .
$$

\section{Springer}

(0) 2013 Bor; licensee Springer. This is an Open Access article distributed under the terms of the Creative Commons Attribution License (http://creativecommons.org/licenses/by/2.0), which permits unrestricted use, distribution, and reproduction in any medium, provided the original work is properly cited. 
In the special case, if we take $\varphi_{n}=n^{1-\frac{1}{k}}$, then $\varphi-|C, \alpha|_{k}$ summability is the same as $|C, \alpha|_{k}$ summability (see [4]). Also, if we take $\varphi_{n}=n^{\delta+1-\frac{1}{k}}$, then $\varphi-|C, \alpha|_{k}$ summability reduces to $|C, \alpha ; \delta|_{k}$ summability (see [5]).

\section{Known result}

In [6], we have proved the following theorem.

Theorem A Let $\left(\lambda_{n}\right) \in \mathcal{B V}$ and let $\left(X_{n}\right)$ be a quasi- $\sigma$-power increasing sequence for some $\sigma(0<\sigma<1)$. Suppose also that there exist sequences $\left(\beta_{n}\right)$ and $\left(\lambda_{n}\right)$ such that

$$
\begin{aligned}
& \left|\Delta \lambda_{n}\right| \leq \beta_{n}, \\
& \beta_{n} \rightarrow 0 \quad \text { as } n \rightarrow \infty, \\
& \sum_{n=1}^{\infty} n\left|\Delta \beta_{n}\right| X_{n}<\infty, \\
& \left|\lambda_{n}\right| X_{n}=O(1) \text { as } n \rightarrow \infty .
\end{aligned}
$$

If there exists an $\epsilon>0$ such that the sequence $\left(n^{\epsilon-k}\left|\varphi_{n}\right|^{k}\right)$ is non-increasing and if the sequence $\left(w_{n}^{\alpha}\right)$ defined by (see [7])

$$
w_{n}^{\alpha}= \begin{cases}\left|t_{n}^{\alpha}\right|, & \alpha=1, \\ \max _{1 \leq v \leq n}\left|t_{v}^{\alpha}\right|, & 0<\alpha<1,\end{cases}
$$

satisfies the condition

$$
\sum_{n=1}^{m} \frac{\left(\left|\varphi_{n}\right| w_{n}^{\alpha}\right)^{k}}{n^{k}}=O\left(X_{m}\right) \quad \text { as } m \rightarrow \infty
$$

then the series $\sum a_{n} \lambda_{n}$ is summable $\varphi-|C, \alpha|_{k}, k \geq 1,0<\alpha \leq 1$ and $k \alpha+\epsilon>1$.

It should be remarked that we have added the condition ' $\left(\lambda_{n}\right) \in \mathcal{B V}$ ' in the statement of Theorem A because it is necessary.

\section{The main result}

The aim of this paper is to prove Theorem A under less and weaker conditions. Now, we will prove the following theorem.

Theorem Let $\left(X_{n}\right)$ be a quasi- $\sigma$-power increasing sequence for some $\sigma(0<\sigma<1)$. If there exists an $\epsilon>0$ such that the sequence $\left(n^{\epsilon-k}\left|\varphi_{n}\right|^{k}\right)$ is non-increasing and if the conditions from (5) to (8) are satisfied and if the condition

$$
\sum_{n=1}^{m} \frac{\left(\left|\varphi_{n}\right| w_{n}^{\alpha}\right)^{k}}{n^{k} X_{n}^{k-1}}=O\left(X_{m}\right) \quad \text { as } m \rightarrow \infty
$$

is satisfied, then the series $\sum a_{n} \lambda_{n}$ is summable $\varphi-|C, \alpha|_{k}, k \geq 1,0<\alpha \leq 1$, and $k(\alpha-1)+$ $\epsilon>1$. 
Remark It should be noted that condition (11) is the same as condition (10) when $k=1$. When $k>1$, condition (11) is weaker than condition (10), but the converse is not true. As in [8] we can show that if (10) is satisfied, then we get that

$$
\sum_{n=1}^{m} \frac{\left(\left|\varphi_{n}\right| w_{n}^{\alpha}\right)^{k}}{n^{k} X_{n}^{k-1}}=O\left(\frac{1}{X_{1}^{k-1}}\right) \sum_{n=1}^{m} \frac{\left(\left|\varphi_{n}\right| w_{n}^{\alpha}\right)^{k}}{n^{k}}=O\left(X_{m}\right) .
$$

If (11) is satisfied, then for $k>1$ we obtain that

$$
\sum_{n=1}^{m} \frac{\left(\left|\varphi_{n}\right| w_{n}^{\alpha}\right)^{k}}{n^{k}}=\sum_{n=1}^{m} X_{n}^{k-1} \frac{\left(\left|\varphi_{n}\right| w_{n}^{\alpha}\right)^{k}}{n^{k} X_{n}^{k-1}}=O\left(X_{m}^{k-1}\right) \sum_{n=1}^{m} \frac{\left(\left|\varphi_{n}\right| w_{n}^{\alpha}\right)^{k}}{n^{k} X_{n}^{k-1}}=O\left(X_{m}^{k}\right) \neq O\left(X_{m}\right) .
$$

Also, it should be noted that the condition ' $\left(\lambda_{n}\right) \in \mathcal{B} \mathcal{V}$ ' has been removed.

We need the following lemmas for the proof of our theorem.

Lemma 1 [9] If $0<\alpha \leq 1$ and $1 \leq v \leq n$, then

$$
\left|\sum_{p=0}^{v} A_{n-p}^{\alpha-1} a_{p}\right| \leq \max _{1 \leq m \leq \nu}\left|\sum_{p=0}^{m} A_{m-p}^{\alpha-1} a_{p}\right| .
$$

Lemma 2 [2] Under the conditions on $\left(X_{n}\right),\left(\beta_{n}\right)$ and $\left(\lambda_{n}\right)$, as expressed in the statement of the theorem, we have the following:

$$
\begin{aligned}
& n \beta_{n} X_{n}=O(1) \quad \text { as } n \rightarrow \infty, \\
& \sum_{n=1}^{\infty} \beta_{n} X_{n}<\infty .
\end{aligned}
$$

\section{Proof of the theorem}

Let $\left(T_{n}^{\alpha}\right)$ be the $n$th $(C, \alpha)$, with $0<\alpha \leq 1$, mean of the sequence $\left(n a_{n} \lambda_{n}\right)$. Then, by (2), we have

$$
T_{n}^{\alpha}=\frac{1}{A_{n}^{\alpha}} \sum_{v=1}^{n} A_{n-\nu}^{\alpha-1} v a_{v} \lambda_{v}
$$

First, applying Abel's transformation and then using Lemma 1, we get that

$$
\begin{aligned}
T_{n}^{\alpha}= & \frac{1}{A_{n}^{\alpha}} \sum_{v=1}^{n-1} \Delta \lambda_{v} \sum_{p=1}^{v} A_{n-p}^{\alpha-1} p a_{p}+\frac{\lambda_{n}}{A_{n}^{\alpha}} \sum_{v=1}^{n} A_{n-\nu}^{\alpha-1} v a_{v}, \\
\left|T_{n}^{\alpha}\right| & \leq \frac{1}{A_{n}^{\alpha}} \sum_{v=1}^{n-1}\left|\Delta \lambda_{v}\right|\left|\sum_{p=1}^{v} A_{n-p}^{\alpha-1} p a_{p}\right|+\frac{\left|\lambda_{n}\right|}{A_{n}^{\alpha}}\left|\sum_{v=1}^{n} A_{n-\nu}^{\alpha-1} v a_{v}\right| \\
& \leq \frac{1}{A_{n}^{\alpha}} \sum_{v=1}^{n-1} A_{v}^{\alpha} w_{v}^{\alpha}\left|\Delta \lambda_{v}\right|+\left|\lambda_{n}\right| w_{n}^{\alpha} \\
& =T_{n, 1}^{\alpha}+T_{n, 2}^{\alpha} .
\end{aligned}
$$


To complete the proof of the theorem, by Minkowski's inequality, it is sufficient to show that

$$
\sum_{n=1}^{\infty} n^{-k}\left|\varphi_{n} T_{n, r}^{\alpha}\right|^{k}<\infty \quad \text { for } r=1,2
$$

Now, when $k>1$, applying Hölder's inequality with indices $k$ and $k^{\prime}$, where $\frac{1}{k}+\frac{1}{k^{\prime}}=1$, we get that

$$
\begin{aligned}
& \sum_{n=2}^{m+1} n^{-k}\left|\varphi_{n} T_{n, 1}^{\alpha}\right|^{k} \leq \sum_{n=2}^{m+1} n^{-k}\left(A_{n}^{\alpha}\right)^{-k}\left|\varphi_{n}\right|^{k}\left\{\sum_{v=1}^{n-1} A_{v}^{\alpha} w_{v}^{\alpha}\left|\Delta \lambda_{v}\right|\right\}^{k} \\
& \leq \sum_{n=2}^{m+1} n^{-k} n^{-\alpha k}\left|\varphi_{n}\right|^{k} \sum_{v=1}^{n-1} v^{\alpha k}\left(w_{v}^{\alpha}\right)^{k}\left|\Delta \lambda_{v}\right|^{k} \times\left\{\sum_{v=1}^{n-1} 1\right\}^{k-1} \\
& =O(1) \sum_{v=1}^{m} v^{\alpha k}\left(w_{v}^{\alpha}\right)^{k}\left(\beta_{v}\right)^{k} \sum_{n=v+1}^{m+1} \frac{n^{\epsilon-k}\left|\varphi_{n}\right|^{k}}{n^{k(\alpha-1)+\epsilon+1}} \\
& =O(1) \sum_{v=1}^{m} v^{\alpha k}\left(w_{v}^{\alpha}\right)^{k} \beta_{v}\left(\beta_{v}\right)^{k-1} v^{\epsilon-k}\left|\varphi_{v}\right|^{k} \sum_{n=v+1}^{m+1} \frac{1}{n^{k(\alpha-1)+\epsilon+1}} \\
& =O(1) \sum_{v=1}^{m} v^{\alpha k}\left(w_{v}^{\alpha}\right)^{k} \beta_{v}\left(\beta_{v}\right)^{k-1} v^{\epsilon-k}\left|\varphi_{v}\right|^{k} \int_{v}^{\infty} \frac{d x}{x^{k(\alpha-1)+\epsilon+1}} \\
& =O(1) \sum_{v=1}^{m} \beta_{v}\left(\beta_{v}\right)^{k-1}\left(w_{v}^{\alpha}\left|\varphi_{v}\right|\right)^{k} \\
& =O(1) \sum_{v=1}^{m} \beta_{v}\left(\frac{1}{v X_{v}}\right)^{k-1}\left(w_{v}^{\alpha}\left|\varphi_{v}\right|\right)^{k} \\
& =O(1) \sum_{v=1}^{m-1} \Delta\left(v \beta_{v}\right) \sum_{r=1}^{v} \frac{\left(\left|\varphi_{r}\right| w_{r}^{\alpha}\right)^{k}}{r^{k} X_{r}^{k-1}}+O(1) m \beta_{m} \sum_{v=1}^{m} \frac{\left(\left|\varphi_{v}\right| w_{v}^{\alpha}\right)^{k}}{v^{k} X_{v}^{k-1}} \\
& =O(1) \sum_{v=1}^{m-1}\left|\Delta\left(v \beta_{v}\right)\right| X_{v}+O(1) m \beta_{m} X_{m} \\
& =O(1) \sum_{v=1}^{m-1}\left|(v+1) \Delta \beta_{v}-\beta_{v}\right| X_{v}+O(1) m \beta_{m} X_{m} \\
& =O(1) \sum_{v=1}^{m-1} v\left|\Delta \beta_{v}\right| X_{v}+O(1) \sum_{v=1}^{m-1} \beta_{v} X_{v}+O(1) m \beta_{m} X_{m} \\
& =O(1) \quad \text { as } m \rightarrow \infty
\end{aligned}
$$

by virtue of the hypotheses of the theorem and Lemma 2. Finally, we have that

$$
\begin{aligned}
\sum_{n=1}^{m} n^{-k}\left|\varphi_{n} T_{n, 2}^{\alpha}\right|^{k} & =\sum_{n=1}^{m}\left|\lambda_{n}\right|\left|\lambda_{n}\right|^{k-1} n^{-k}\left(w_{n}^{\alpha}\left|\varphi_{n}\right|\right)^{k} \\
& =O(1) \sum_{n=1}^{m}\left|\lambda_{n}\right|\left(\frac{1}{X_{n}}\right)^{k-1} n^{-k}\left(w_{n}^{\alpha}\left|\varphi_{n}\right|\right)^{k}
\end{aligned}
$$




$$
\begin{aligned}
& =O(1) \sum_{n=1}^{m-1} \Delta\left|\lambda_{n}\right| \sum_{n=1}^{m} \frac{\left(\left|\varphi_{n}\right| w_{n}^{\alpha}\right)^{k}}{n^{k} X_{n}^{k-1}}+O(1)\left|\lambda_{m}\right| \sum_{n=1}^{m} \frac{\left(\left|\varphi_{n}\right| w_{n}^{\alpha}\right)^{k}}{n^{k} X_{n}^{k-1}} \\
& =O(1) \sum_{n=1}^{m-1}\left|\Delta \lambda_{n}\right| X_{n}+O(1)\left|\lambda_{m}\right| X_{m} \\
& =O(1) \sum_{n=1}^{m-1} \beta_{n} X_{n}+O(1)\left|\lambda_{m}\right| X_{m}=O(1) \quad \text { as } m \rightarrow \infty
\end{aligned}
$$

by virtue of the hypotheses of the theorem and Lemma 2 . This completes the proof of the theorem. If we take $\epsilon=1$ and $\varphi_{n}=n^{1-\frac{1}{k}}$ (resp. $\epsilon=1, \alpha=1$ and $\varphi_{n}=n^{1-\frac{1}{k}}$ ), then we get a new result dealing with $|C, \alpha|_{k}$ (resp. $|C, 1|_{k}$ ) summability factors. Also, if we set $\epsilon=1$ and $\varphi_{n}=n^{\delta+1-\frac{1}{k}}$, then we get another new result concerning the $|C, \alpha ; \delta|_{k}$ summability factors. Finally, if we take $\left(X_{n}\right)$ as an almost increasing sequence, then we get the result of Bor and Seyhan under weaker conditions (see [10]).

\section{Competing interests}

The author declares that he has no competing interests.

\section{Acknowledgements}

Dedicated to Professor Hari M Srivastava.

\section{Received: 22 December 2012 Accepted: 9 February 2013 Published: 26 February 2013}

\section{References}

1. Bari, NK, Stečkin, SB: Best approximation and differential properties of two conjugate functions. Tr. Mosk. Mat. Obŝ. 5, 483-522 (1956) (in Russian)

2. Leindler, L: A new application of quasi power increasing sequences. Publ. Math. (Debr.) 58, 791-796 (2001)

3. Balcı, M: Absolute $\varphi$-summability factors. Commun. Fac. Sci. Univ. Ankara, Ser. A1 29, 63-80 (1980)

4. Flett, TM: On an extension of absolute summability and some theorems of Littlewood and Paley. Proc. Lond. Math. Soc. 7, 113-141 (1957)

5. Flett, TM: Some more theorems concerning the absolute summability of Fourier series. Proc. Lond. Math. Soc. 8 , 357-387 (1958)

6. Bor, H, Özarslan, HS: A study on quasi power increasing sequences. Rocky Mt. J. Math. 38, 801-807 (2008)

7. Pati, T: The summability factors of infinite series. Duke Math. J. 21, 271-284 (1954)

8. Sulaiman, WT: On a new application of almost increasing sequences. Bull. Math. Anal. Appl. 4(3), 29-33 (2012)

9. Bosanquet, LS: A mean value theorem. J. Lond. Math. Soc. 16, 146-148 (1941)

10. Bor, H, Seyhan, H: A note on almost increasing sequences. Comment. Math. Prace Mat. 39, 37-42 (1999)

doi:10.1186/1029-242X-2013-69

Cite this article as: Bor: A new application of quasi power increasing sequences. I. Journal of Inequalities and Applications 2013 2013:69.

\section{Submit your manuscript to a SpringerOpen ${ }^{\circ}$ journal and benefit from:}

- Convenient online submission

- Rigorous peer review

- Immediate publication on acceptance

- Open access: articles freely available online

- High visibility within the field

- Retaining the copyright to your article 